\title{
Treatment challenges in and outside a network setting: Head and neck cancers
}

\author{
Ester Orlandi ${ }^{\text {a, }}{ }^{*}, 1$, Salvatore Alfieri ${ }^{\text {b, }}{ }^{1}$, Christian Simon ${ }^{c}$, Annalisa Trama ${ }^{\mathrm{d}}$, Lisa Licitra ${ }^{\mathrm{b}, \mathrm{e}}$, \\ RARECAREnet Working Group ${ }^{2}$
}

${ }^{a}$ Radiotherapy 2 Unit, Fondazione IRCCS Istituto Nazionale dei Tumori, Milan, Italy

${ }^{\mathrm{b}}$ Medical Oncology 3 Department, Fondazione IRCCS Istituto Nazionale dei Tumori, Milan, Italy

${ }^{\mathrm{c}}$ Department of Otolaryngology Head and Neck Surgery, CHUV University of Lausanne, Switzerland

${ }^{\mathrm{d}}$ Evaluative Epidemiology Unit, Fondazione IRCCS Istituto Nazionale dei Tumori, Milan, Italy

e University of Milan, Italy

\section{A R T I C L E I N F O}

\section{Article history:}

Accepted 6 February 2018

Available online $\mathrm{xxx}$

\section{Keywords:}

Head and neck cancer

Rare disease

Multidisciplinary team

European reference networks joint action of rare cancers

\begin{abstract}
A B S T R A C T
Head and neck cancer (HNC) is a rare disease that can affect different sites and is characterized by variable incidence and 5-year survival rates across Europe.

Multiple factors need to be considered when choosing the most appropriate treatment for HNC patients, such as age, comorbidities, social issues, and especially whether to prefer surgery or radiationbased protocols. Given the complexity of this scenario, the creation of a highly specialized multidisciplinary team is recommended to guarantee the best oncological outcome and prevent or adequately treat any adverse effect.

Data from literature suggest that the multidisciplinary team-based approach is beneficial for HNC patients and lead to improved survival rates. This result is likely due to improved diagnostic and staging accuracy, a more efficacious therapeutic approach and enhanced communication across disciplines. Despite the benefit of MTD, it must be noted that this approach requires considerable time, effort and financial resources and is usually more frequent in highly organized and high-volume centers. Literature data on clinical research suggest that patients treated in high-accrual centers report better treatment outcomes compared to patients treated in low-volume centers, where a lower radiotherapy-compliance and worst overall survival have been reported.

There is general agreement that treatment of rare cancers such as HNC should be concentrated in high volume, specialized and multidisciplinary centers. In order to achieve this goal, the creation of international collaboration network is fundamental. The European Reference Networks for example aim to create an international virtual advisory board, whose objectives are the exchange of expertise, training, clinical collaboration and the reduction of disparities and enhancement of rationalize migration across Europe.

The purpose of our work is to review all aspects and challenges in and outside this network setting planned for the management of HNC patients.
\end{abstract}

(๑) 2018 Elsevier Ltd, BASO The Association for Cancer Surgery, and the European Society of Surgical Oncology. All rights reserved.

\footnotetext{
* Corresponding author. Radiotherapy 2 Unit, Fondazione IRCCS Istituto Nazionale dei Tumori, Via Giacomo Venezian, 1, 20133, Milan, Italy.

E-mail address: ester.orlandi@istitutotumori.mi.it (E. Orlandi).

${ }^{1}$ Equal Contributors.

2 RARECAREnet Working Group collab authors are listed in appendix.
}

\section{Introduction}

Head and neck cancer (HNC) represents a group of rare oncological diseases with individual and specific demographic characteristics, variable incidences and 5-year survival rates across Europe.

In this paper we discuss several aspects of the (HNC) management, focusing on the role and influence of networking in this rare kind of cancer, in order to achieve better results in clinical and 
research fields. In detail, the manuscript consists of three major parts: first, we presented a summary of epidemiological data on HNC, reporting the differences in terms of incidence rates across Europe during the last decade. Second, we focus our attention on HNC management, summarizing the most important treatment options and highlighting the main challenges of a multidisciplinary approach, which has already been reported as beneficial for HNC patients. We also briefly discuss the differences between high versus low-volume accrual centers and the most critical issues of multidisciplinary context, both in clinical practice and in the research setting. Last of all, we describe another side of the networking in HNC throughout the experience of the European Reference Networks, a virtual network connecting several healthcare providers across Europe, and the Joint Action of Rare Cancers, aimed at advancing quality of care and research in rare cancers.

\section{Epidemiology of head and neck cancers}

HNC include a group of neoplasms of various anatomic sites different in terms of aetiology, diagnostic and treatment approaches. HNCs are typically cancers that develop in smokers and in a population with large alcohol consumption [1]. Other risk factors are viral infection (Epstein-Barr Virus (EBV) for nasopharyngeal cancer [2] and Human Papilloma Virus (HPV) for oropharynx cancer [3]), occupational exposure [4], radiation for major and minor salivary gland cancers [5].

According to the project RARECAREnet (information network for rare cancers www.rarecarenet.eu), HNCs are comprised of epithelial tumours of the nasal cavity and sinuses, nasopharynx, hypopharynx, larynx, oropharynx, oral cavity, lip, eye and its adnexa, middle ear, major salivary glands and, salivary-gland type tumours. The RARECAREnet HNCs list do not include non-epithelial histotypes, such as melanomas. Thus, the results presented should be carefully compared to published figures based on topography only.

The epidemiological indicators reported in this paper are based on the RARECAREnet on-line analysis tool (http://app.rarecarenet. eu/analysis.php). Overall 94 European population-based cancer registries (CRs) adhered to RARECAREnet providing information on cancer patients diagnosed up to 2007 and followed-up for vital status ascertainment to the end of 2008 or later.

All HNCs are rare since the incidence rate (IR) of each of the HNC sub site was $<6 / 100,000$ in the period of diagnosis 2000-2007. However, IR differs among HNC sub sites. The most common were the epithelial tumours of larynx (IR $=4.6 / 100,000 ; 95 \% \mathrm{CI}=$ $4.5-4.6$ ), of oral cavity ( $\mathrm{IR}=3.5 / 100,000 ; 95 \% \mathrm{CI}=3.4-3.5$ ), and oropharynx ( $\mathrm{IR}=3.3 / 100,000 ; 95 \% \mathrm{CI}=3.3-3.5$ ). The rarest were the epithelial tumours of nasal cavity and sinuses ( $I R=0.45$ / $100,000 ; 95 \% \mathrm{CI}=0.44-0.46)$, of nasopharynx ( $\mathrm{IR}=0.47 ; 95 \%$ $\mathrm{CI}=0.46-0.49)$ and of middle ear ( $\mathrm{IR}=0.03 / 100,000 ; 95 \%$ $\mathrm{CI}=0.03-0.04)$. The EUropean reference network on Rare Adults solid CANcers (EURACAN) is working on the rarest HNCs plus the salivary gland tumours of head and neck.

For all HNCs, the highest incidence was observed in patients of 65 years of age and older. For the oropharynx and nasopharynx, IRs were relatively high also for those aged 25-64 years at diagnosis. This could be due to the viral exposure.

While for all sites the incidence between 1995 and 2007 in Europe remained stable or decreased, an increase was observed for oropharyngeal cancer from $2.3(95 \% \mathrm{CI}=2.2-2.3)$ in the period $1995-1998$ to $2.9(95 \% \mathrm{CI}=2.8-2.9)$ in the period $2003-2007$. Interesting to note that also the 5-year relative survival (RS) for oropharyngeal cancer increased from $37 \%(95 \% \mathrm{CI}=36-38)$ in 1999 to $44 \%(95 \% \mathrm{CI}=43-345)$ in 2007 . The larger proportion of less aggressive tumours attributed to HPV might have influenced incidence increase and survival gain.
There was a geographical variation in incidence for epithelial tumours of nasopharynx, hypopharynx, oropharynx, larynx and oral cavity. Southern Europe experienced the highest IR for epithelial tumours of nasopharynx and larynx. Central Europe presented the highest IR epithelial tumours of the oral cavity, hypopharynx and oropharynx. The IR for epithelial tumours of oral cavity, oropharynx, larynx, and hypopharynx were lower in UK and Ireland and Northern Europe. Geographical differences in incidence, as well as differences between men and women, may be explained by differences in risk factors between the two sexes.

The epithelial tumours with the highest 5-year RS in Europe (period of diagnosis 2000-2007) were in descending order epithelial tumours of eye and adnexa $81 \%(95 \% \mathrm{CI}=76-86)$, salivary gland type tumours of head and neck $67 \%(95 \% \mathrm{CI}=66-69)$, epithelial tumours of major salivary glands $1 \%(95 \% \mathrm{CI}=60-62)$ and of larynx $61 \%(95 \% \mathrm{CI}=60-61)$. All the other sub sites had 5-year RS lower than $50 \%$ but lips. Epithelial tumours of hypopharynx were those with the lowest 5-year RS 25\% (95\%CI = 24-26). Thus, EURACAN includes most of the rarest HNCs which correspond also to most of those with low survival $(<50 \%)$.

The 5-year RS varied also by geographic area. Easter Europe had the lowest 5-year RS for each of the HNC considered. Northern Europe had the highest 5-year RS for epithelial tumours of oropharynx and central Europe for hypopharynx. Southern Europe had the lowest 5-year RS (not considering eastern Europe) for the epithelial tumours of oral cavity and oropharynx. UK and Ireland had slightly lower 5-year RS (excluding eastern Europe) for nasopharynx and hypopharynx. Differences in survival might be explained considering the different distribution of risk factors which are also prognostic factors such as smoking and alcohol, to different socio-economic status, to different health care organisation impacting on the management and quality of care of HNCs.

\section{Multidisciplinary care in the treatment of head and neck cancers}

Several factors make treatment decisions in HNC very challenging [6]. Other than histology, sub-site and staging, even age and sex, concomitant comorbidities and social issues (i.e. the presence or absence of family caregivers) are all aspects that need to be strictly considered when HNC patients are managed. For example, oral cavity cancer patients are often elderly with remarkable medical comorbidities and, as a consequence of the close proximity of functionally important anatomical areas, it is very difficult to predict side effects of therapy. Instead, nasopharyngeal and HPV positive cancer patients are frequently younger with limited concomitant conditions. These patients have a longer life expectancy requiring a careful evaluation of potential reactions with an appropriate management.

In addition to this, there is almost complete lack of level I evidence to guide choices between surgery and radiation-based protocols. However, certain HNCs are typically managed by head and neck surgeons, i.e. oral cavity cancer, whilst other tumours, such as nasopharyngeal cancers, are primarily loco regionally treated with radiotherapy (RT).

In general, early-stage laryngeal and pharyngeal cancer patients can be successfully managed with single modality therapy, either RT or surgery, in a high percentage of cases (80\%-90\%). Instead, locally advanced disease needs to be cured with multimodality approaches including surgery followed by RT with or without chemotherapy (CHT), in case of adverse pathological features, or chemoradiotherapy (CRT) up front, thus achieving, 5-year overall survival (OS) rate of 40\%-50\% [7-9]. Organ-preservation approaches combining chemo- and RT are generally favored, with similar outcome results compared to a laryngectomy [10], 
especially induction chemotherapy followed by RT [11,12]. Recently, for oropharyngeal cancers, minimally invasive surgical options with or without neck dissection and risk-stratified adjuvant treatment, have prospectively shown clinical results comparable with CRT [13-16] for both HPV-positive and HPV-negative tumours, although comparative efficacy studies are lacking [17,18].

Therefore, HNC management requires a highly specialized multidisciplinary team (MDT) to guarantee the best oncological outcome and prevent and adequately treat any adverse effect of treatment by using coordinated professional efforts. Indeed, a balance between cure and complications remains the primary challenge, requiring optimization of the therapeutic effect with prudent and individualized application of the various treatment modalities in appropriately selected patients [19].

Evidence accumulated over the last years suggests that such MDTs are beneficial for HNC patients. An Australian study showed improved survival in stage IV HNC patients whose cases were discussed at multidisciplinary meetings. The authors concluded that the added value for MDT-managed HNC patients was properly a higher use of multimodality treatment strategy and the presence of multiple providers influencing decision-making in a MDT context [20]. Moreover, this holds particularly true for HNC patients treated with surgery up-front. In a nationwide cohort study on 16,991 patients afflicted with oral cavity cancers, patients treated within the framework of MDTs had better OS rates [Hazard Ratio (HR) 0,94; CI $=0.89-1.00]$. In particular patients with stage IV disease, males, and patients with higher Charlson Comorbidity Index (between 4 and 6) profited the most [21]. However, we cannot exclude the migration bias might have affected this study, in which more accurate staging procedures makes the group of multidisciplinary IV disease less advanced than that of the non multidisciplinary IV stage cancer, which suffers of a less accurate staging. The improved outcome is likely a consequence of improved diagnostic and staging accuracy and/or a more efficacious therapeutic approach as well as a better communication across disciplines enabled by the MDT. After MDT meetings, higher rates of dental assessment (59\% versus $22 \%, \mathrm{p}<0.0001$ ), nutritional assessment (57\% versus $39 \%$, $\mathrm{p}=0.015$ ), positron emission tomography (PET) staging (41\% versus $2 \%, \mathrm{p}<0.0001$ ), CRT for locally advanced disease (66\% versus $16 \%$, $\mathrm{p}<0.0001)$ and use of adjuvant CRT for high risk disease $(49 \%$ versus $16 \%, \mathrm{p}<0.0001$ ) were found. MDT meetings were reported to lead to a staging refinement and greater employment of more aggressive therapies (higher radiation dose, integration of more treatment approaches) [22-24]. In particular, a change in diagnostic and therapeutic approaches was discovered for rare tumours, such as paranasal sinuses cancer, nasopharyngeal cancer and salivary gland carcinoma [24]. In another study, MDTs significantly improved timing from initial referral to first otolaryngologist visit (27.5-16.5 days, $\mathrm{p}<0.0001)$ and from a diagnostic positive biopsy to the start of definitive treatment (35-27 days, $\mathrm{p}=0.04$ ) [25].

A MDT usually involves a centralization of care with specialization, but it also takes considerable time, effort and financial resources. For all of these reasons, it better works in high volume and referral cancer centers even if the cut-off used to define the case volume as "high" or "low" is still to be established [26].

However, cancer treatment experience concerns the overall quality of all oncological disciplines involved.

From a surgical point of view, the matter of influence of surgeon and/or hospital case volume on the HNC outcomes is far to be stated [26] because of lack of studies analysing both of these two factors concomitantly. Nevertheless, bearing in mind the importance of expertise of each surgeon, it should be also considered that hospital volume plays a pivotal role. In fact, surgery of HNC patients usually requires such specific issues differing from those needed in other kind of surgery: typical long-stay of HNC patients after surgery due to peri operative complications involving several healthcare professionals.

From a radiotherapeutic point of view, different aspects may potentially explain the difference in outcomes in the high-volume context. It is important to consider the expertise in prescribing and interpreting staging studies, in delineating both target volumes and normal areas included in irradiated volumes; but also the consolidated organization and use of a comprehensive quality assurance (QA) program for all RT procedures to ensure safe and effective treatment delivery. It is needed to evaluate the doseintensity of RT and CHT, as well as the use of multidisciplinary cooperation and/or better expertise of treating physicians in supportive care (regarding nutritional and infectious issues, preemptive swallowing exercises, pain treatment) to reduce the risk of treatment discontinuation due to toxicity and acute mortality [19].

Regarding the role of medical oncologists in the MDT context, the European Society for Medical Oncology (ESMO) has clearly stated in a position paper published in 2014 the importance of the oncologists as "core-members" of MDTs [27]. Recently, ESMO confirmed its great interest in this field considering the MDT work as crucial part to better train young oncologists [28] and fellows [29], thus becoming one of the key recommendations of the ESMO/ ASCO for a global curriculum in medical oncology [29]. A medical oncologist working well in the context of an MDT could be of benefit for the patient, by offering the best systemic (standard and/ or experimental) treatment options and, at the same time, exploiting his own internal medicine skills and capability of general clinical overview.

\section{Multidisciplinary care and case volume in clinical research}

Exploring beyond the clinical practice, the benefit of case volume has also been assessed in the clinical research field. Specifically, Wuthrick EJ et al. [30] reported worsening in 5-year locoregional failure ( $21 \%$ vs $36 \%$ ) and 5 -year OS (51\% vs $69 \%$ ) for oropharyngeal cancer patients treated in the Radiation Therapy Oncology Group (RTOG) 0129 randomized trial, if analysed as patients enrolled within historically low accrual centers (HLACs) vs historically high accrual centers (HHACs), respectively. To evaluate the accrual rate and differentiate between HLACs and HHACs, the authors reviewed how many HNC patients were enrolled from 1997 to 2002 in RTOG trials by each of 303 considered centers. An accrual of at least 42 patients was considered to define the centers as HHACs. Among the 471 patients enrolled in the RTOG 0129 study, the majority of them (321) came from HLACs. After adjusting for age, $\mathrm{T}$ and $\mathrm{N}$ classification, performance status, smoking and HPV status, the risk of death increased of $91 \%$ for patients treated at HLACs, who also were less compliant with the planned RT treatment compared with those coming from HHACs ( $11 \%$ vs $5 \%$ of RT non-compliance). A positive relationship between high volume recruitment and treatment outcome was also demonstrated by the Trans Tasman Radiation Oncology Group (TROG) in TROG 02.02 study [31]. Person LJ et al. reported that centers with low accrual ( $<5$ patients/year) had significantly lower RT compliance compared with those with higher accrual ( $>20$ patients). This discrepancy resulted in a higher 2 -year locoregional failure rate (46\% vs $22 \%$ ) and worse 2 -year OS (50\% vs $70 \%$ ) for the lowest enrolling centers. These data [30,31] confirm the importance of caseload in the clinical trial setting, where expertise and multidisciplinary care are strictly advocated. Multidisciplinarity may also lead to an increase of "surgical" and "integrated" studies. The latter regards those trials designed and performed thanks to cooperation of multiple professionals and/or centers involved in the HNC patients' care [32]. 
This brings us to another aspect of multidisciplinarity and clinical trials: certain investigators of clinical multidisciplinary trials may not be sufficiently competent, based on their clinical training and experience, to run such trials. A formal training and certificate would seem warranted. Having said that, none of these measures should prevent multidisciplinary trials in the field of HNC to be conducted.

\section{Relevance and role of the European reference networks (ERN)}

In order to improve the management of complex and rare diseases, European reference networks (ERNs), the virtual networks bringing together healthcare providers across Europe, were created. The concept is the establishment of virtual advisory boards across medical specialties typically involved with the rare disease using dedicated IT-platforms and tools. In March 2017 the European Commission created 24 of these networks; among them three networks concerned with rare cancers (ERN EURACAN, ERN EuroBloodNet and ERN PaedCan). These three networks cover rare solid adult cancers (ERN EURACAN), rare hematological diseases (ERN EuroBloodNet) and pediatric cancers (ERN PaedCan). HNCs are represented in EURACAN, which is divided in 10 domains, one per each of the family of rare solid cancers; HNCs are one of the EURACAN domains, specifically G7.

The ERNs should also reduce disparities and rationalize migration from one country to another. Outcome for HNC largely varied across Europe with the lowest 5-year survival for all epithelial HNC in the Eastern European countries. The gap was very big for oral cavity, oropharynx and salivary gland type tumours of the HN area [33]. Among the objectives of the ERNs are the exchange of expertise training, and increase of clinical research collaboration.

As mentioned above, there is general agreement that treatment of rare cancers should be concentrated in high volume, specialized and multidisciplinary centers, and that international collaboration is needed for research for these rare cancers. However, centralization of rare cancer treatments at the population level, measured in seven European countries for the period 2000-2007, seems to greatly vary [34]. Fig. 1 shows that $75 \%$ of treatments were given in 29 hospitals in Belgium, but in only 2 hospitals in Slovenia and Navarra. The mean annual number of treatments provided by hospitals, covering the $75 \%$ of treatments, ranged between 201 and
266 in the Netherlands and Slovenia respectively, but was found to be only 63 in Ireland (Fig. 1). It is certainly to be considered that the number of cases treated by each center depends on population size, rare cancer incidence, and hospital capacity/qualification. Fig. 2 shows the mean admission number [34] per hospital by country for HN epithelial cancers. These estimations [34], which are not influenced by the size of the population, confirm that centralization of the HN treatment varies widely between countries and was highest in Slovenia and the Netherlands.

\section{Relevance and function of the joint action of rare cancers (JARC)}

The JARC is the first joint action on rare cancers. This is important because so far there have been Joint Actions on rare diseases and on cancers but never on rare cancers. Thus, the JARC confirms that the European Union (EU) is starting to prioritize rare cancers perse.

The JARC aims to integrate and maximize efforts of the EU Commission, EU Member States and all stakeholders to advance quality of care and research on rare cancers.

The public health challenge posed by rare cancers combines both the typical problems of rare diseases (such as the limited professional expertise available in the community, or the difficulties in clinical research) and those of cancer, with the need of a timely and appropriate diagnosis and optimal treatment from the very beginning of the patient's journey. An accurate clinical, pathologic and biological assessment of the disease of the individual patient is key to survival and cure, as well as an expert clinical decision provided by a multidisciplinary team. To this end, proper referral of patients and effective clinical networking are crucial in rare cancers.

This is the main reason why JARC decided to shape its efforts, in essence, around the ERNs. The formal activation of ERNs is a cornerstone in the EU cooperation on rare cancers, and this Joint Action should be instrumental to make them grow the best way possible. In fact, JARC aims at optimizing the process of creation of the ERNs, by providing them with operational solutions and professional guidance in the areas of quality of care, epidemiology, research and innovation, education and state of the art definition on prevention, diagnosis and treatment of rare cancers.

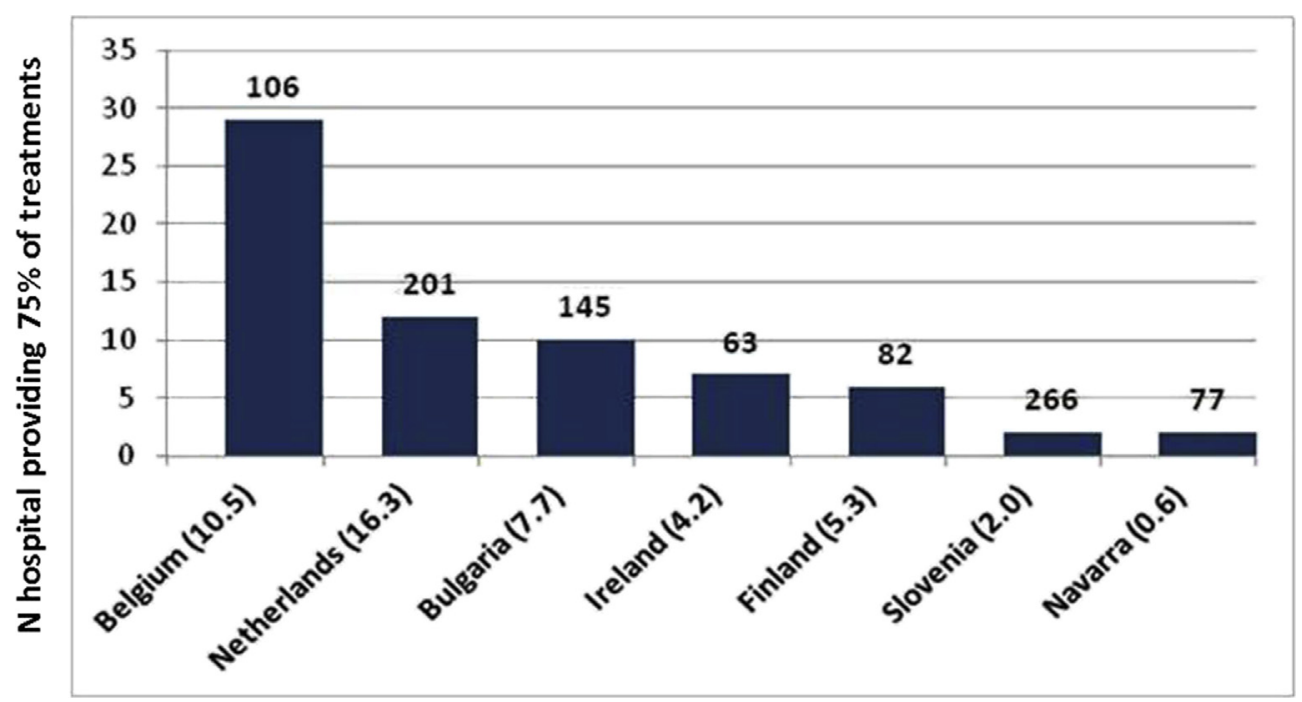

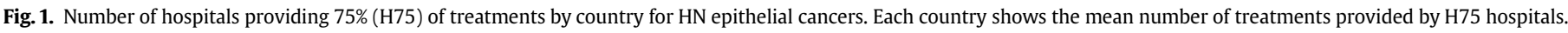
Each country shows the size of population in millions; in brackets on the $\mathrm{X}$ axis. 
Mean admission volume by country for $H \& N$

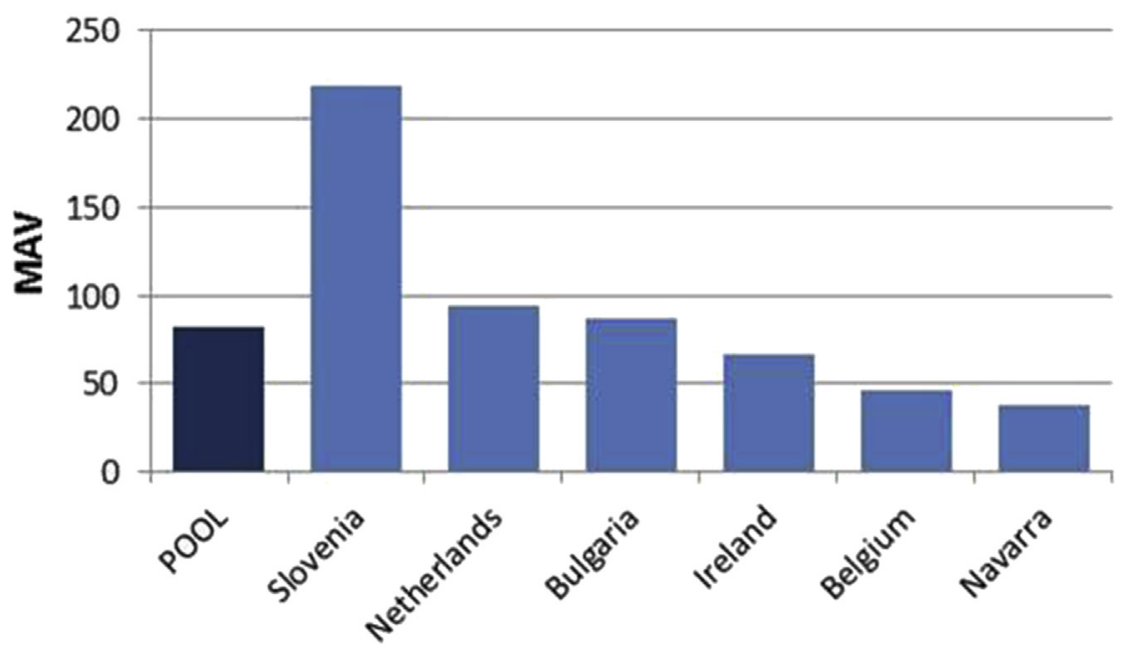

Fig. 2. Mean admission volume (MAV) by country.

\section{Conclusions}

HNC are rare and complex to be managed. Starting from that, multidisciplinarity and case volume (either related to each healthcare professional and facility involved) are both mandatory to achieve better outcomes in clinical and research field. Considering the need of a simultaneous cooperation, novel networks, such as ERNs, are emerging as essential tools in this working context.

\section{Fundings}

This research was partly funded by the European Commission through the Consumers, Health, Agriculture and Food Executive Agency (Chafea); Grant No. 2000111201; Information network on rare cancers-RARECARENet.

\section{Conflicts of interest}

None.

\section{Acknowledgment}

Editorial assistance for the preparation of this manuscript has been provided by Luca Giacomelli, PhD and Ambra Corti. This assistance was supported by internal funds.

\section{Appendix. RARECARENet Working Group}

Monika Hackl (Austrian National Cancer Registry); Elizabeth Van Eycken, Kris Henau (Belgian Cancer Registry); Nadya Dimitrova (Bulgaria Cancer Registry); (Bulgarian Cancer Registry); Mario Sekerija (Croatian Cancer Registry); Ladislav Dušek (Czech National Cancer Registry); Margit Mägi (Estonian Cancer Registry); Nea Malila, Maarit Leinonen (Finnish Cancer Registry); Michel Velten (Bas Rhin Cancer Registry); Xavier Troussard (Basse Normandie Haematological malignancies Registry); Veronique Bouvier (Calvados Digestive Tract Registry); Anne-Valérie Guizard (Calvados, Registre Gèneral des Tumeurs); Anne-Marie Bouvier (Côte d'Or Digestive Tract Registry, Burgundy); Patrick Arveux (Côte d'Or Gynaecologic Cancer registry); Marc Maynadié (Côte d'Or Haematological Malignancies Cancer Registry); Anne-Sophie Woronoff (Doubs Cancer Registry); Michel Robaszkiewicz (Finistère
Digestive Tract Registry); Isabelle Baldi (Gironde CNS Tumour Registry); Alain Monnereau (Gironde Haematological Malignancies); Brigitte Tretarre (Hérault Cancer Registry); Marc Colonna (Isère Cancer Registry); Florence Molinié (Loire-Atlantique/Vendée Cancer Registry); Simona Bara (Manche Cancer Registry); Claire Schvartz (Marne \& Ardennes, Thyroid); Bénédicte Lapôtre-Ledoux (Somme Cancer Registry); Pascale Grosclaude (Tarn Cancer Registry); Roland Stabenow (Berlin; Brandenburg; Mecklenburg-West Pomerania; Saxony; Saxony-Anhalt; Thüringen Cancer Registry); Sabine Luttmann (Bremen Cancer Registry, Leibniz Institute for Prevention Research and Epidemiology); Alice Nennecke (Hamburg Cancer Registry); Jutta Engel, Gabriele Schubert-Fritschle (Munich Cancer Registry); Jan Heidrich (North Rhine-Westphalia Cancer Registry); Bernd Holleczek (Saarland Cancer Registry); Jón Gunnlaugur Jónasson (Icelandic Cancer Registry); Kerri Clough-Gorr, Harry Comber (National Cancer Registry Ireland); Guido Mazzoleni (Alto Adige Cancer Registry); Adriano Giacomin (Biella Cancer Registry); Antonella Sutera Sardo (Catanzaro Cancer Registry); Alessandro Barchielli (Firenze-Prato Cancer Registry); Diego Serraino (Friuli Venezia Giulia, CRO Aviano National Cancer Institute); Roberta De Angelis, Sandra Mallone, Andrea Tavilla, Daniela Pierannunzio, Silvia Rossi, Mariano Santaquilani, Arnold Knijn (Istituto Superiore di Sanità, Rome); Fabio Pannozzo (Latina Cancer Registry); Valerio Gennaro, Lucia Benfatto (Liguria Mesothelioma Registry); Paolo Ricci (Mantova Cancer Registry); Mariangela Autelitano (Milano Cancer Registry); Gianbattista Spagnoli (Modena Cancer Registry); Mario Fusco (Napoli 3 South Cancer Registry); Mario Usala (Nuoro Cancer Registry); Francesco Vitale (Palermo Cancer Registry); Maria Michiara (Parma Cancer Registry); Rosario Tumino (Ragusa Cancer Registry); Lucia Mangone (Reggio Emilia Cancer Registry); Fabio Falcini (Romagna Cancer Registry); Stefano Ferretti (RT Ferrara Cancer Registry); Rosa Angela Filiberti, Enza Marani (RTR Liguria, IRCCS AOU SM-IST); Arturo Iannelli (Salerno Cancer Registry); Flavio Sensi (Sassari Cancer Registry); Silvano Piffer, Maria Gentilini (Servizio Epidemiologia Clinica e Valutativa, Trento); Anselmo Madeddu, Antonio Ziino (Siracusa Cancer Registry); Sergio Maspero (Sondrio Cancer Registry); Pina Candela (Trapani Cancer Registry); Fabrizio Stracci (Umbria Cancer Registry); Giovanna Tagliabue (Varese Province Cancer Registry, Fondazione IRCCS Istituto Nazionale dei Tumori); Massimo Rugge (Veneto Cancer Registry); Annalisa Trama, Gemma Gatta, Laura Botta, Riccardo Capocaccia (Fondazione IRCCS, National Cancer 
Institute, Milan); Santa Pildava (Latvian Cancer Registry); Giedre Smailyte (Lithuaian Cancer Registry); Neville Calleja (Malta National Cancer Registry, Health Information and Research); Tom Børge Johannesen (Norwegian Cancer Registry); Jadwiga Rachtan (Cracow Cancer Registry); Stanisław Góźdź (Kielce Cancer Registry); Jerzy Błaszczyk, Kamila Kępska (Lower Silesia (Wroclaw); Cancer Registry); Gonçalo Forjaz de Lacerda (Açores Cancer Registry); Maria José Bento (Northern Portugal Cancer Registry); Ana Miranda (Southern Portugal Cancer Registry); Chakameh Safaei Diba (Slovakian National Cancer Registry); Enrique Almar (Albacete. Castilla-La Mancha Cancer Registry); Nerea Larrañaga, Arantza Lopez de Munain (Basque Country Cancer Registry); Ana TorrellaRamos (Castellón-Valencia (breast); Cancer Registry); José María Díaz García (Cuenca Cancer Registry); Rafael Marcos-Gragera (Girona Cancer Registry); Maria Josè Sanchez (Granada Cancer Registry , CIBERESP, ibs.Granada); Carmen Navarro, Diego Salmeron(Murcia Cancer Registry, CIBERESP, IMIB-Arrixaca); Conchi Moreno-Iribas (Navarra Cancer Registry, CIBERESP); Jaume Galceran, Marià Carulla (Tarragona Cancer Registry); Mohsen Mousavi (Basel Cancer Registry); Christine Bouchardy (Geneva Cancer Registry); Silvia M. Ess (Grisons-Glarus, St. Gallen Cancer Registry); Andrea Bordoni (Ticino Cancer Registry); Isabelle Konzelmann (Valais Cancer Registry); Jem Rashbass (Public Health England); Anna Gavin (Northern Ireland Cancer Registry ); David H Brewster (Scotland Cancer Registry); Dyfed Wyn Huws (Welsh Cancer Intelligence and Surveillance Unit); Otto Visser (The Netherlands Cancer Registry); Magdalena Bielska-Lasota (National Institute of Public Health-NIH, Warszawa); Maja Primic-Zakelj (Cancer Registry of Republic of Slovenia); Ian Kunkler (Edinburgh Cancer Centre, University of Edinburgh); Ellen Benhamou (Institut de Cancérologie Gustave Roussy, Villejuif, France).

\section{References}

[1] Lubin JH, Purdue M, Kelsey K, Zhang ZF, Winn D, Wei Q et al. Total exposure and exposure rate effects for alcohol and smoking and risk of head and neck cancer: a pooled analysis of case-control studies. Am J Epidemiol 2009;170: 937-47.

[2] Chang ET, Adami HO. The enigmatic epidemiology of nasopharyngeal carcinoma. Cancer Epidemiol Biomarkers Prev 2006;15:1765-77.

[3] Mork J, Lie AK, Glattre E, Hallmans G, Jellum E, Koskela P, et al. Human papillomavirus infection as a risk factor for squamous-cell carcinoma of the head and neck. N Engl J Med 2001;344:1125-31.

[4] Pukkala E, Martinsen JI, Lynge E, Gunnarsdottir HK, Sparén P, Tryggvadottir L, et al. Occupation and cancer- follow-up of 15 million people in five Nordic countries. Acta Oncol 2009;48:646-790.

[5] Guzzo M, Locati LD, Prott FJ, Gatta G, McGurk M, Licitra L. Major and minor salivary gland tumors. Crit Rev Oncol Hematol 2010;74:134-48.

[6] Sharp L, Lewin F, Hellborg H, Lundgren J, Hemmingsson E, Rutqvist LE. When does my treatment start?-The continuum of care for patients with head and neck cancer. Radiother Oncol 2002;63:293-7.

[7] Saloura V, Langerman A, Rudra S, Chin R, Cohen EE. Multidisciplinary care of the patient with head and neck cancer. Surg Oncol Clin N Am 2013;22: $179-215$.

[8] Blanchard P, Baujat B, Holostenco V, Bourredjem A, Baey C, Bourhis J, et al., $\mathrm{MACH}-\mathrm{CH}$ Collaborative Group. Meta-analysis of chemotherapy in head and neck cancer (MACH-NC): a comprehensive analysis by tumour site. Radiother Oncol 2011;100:33-40.

[9] Cooper JS, Pajak TF, Forastiere AA, Jacobs J, Campbell BH, Saxman SB, et al., Radiation Therapy Oncology Group 9501/Intergroup. Postoperative concurrent radiotherapy and chemotherapy for high-risk squamous-cell carcinoma of the head and neck. N Engl J Med 2004;350:1937-44.

[10] Wolf GT, Fisher SG, Hong WK, Hillman R, Spaulding M, Laramore GE, et al. Induction chemotherapy plus radiation compared with surgery plus radiation in patients with advanced laryngeal cancer. N Engl J Med 1991;324:1685-90.

[11] Janoray G, Pointreau Y, Garaud P, Chapet S, Alfonsi M, Sire C, et al. Long-term results of a multicenter randomized Phase III trial of induction chemotherapy with cisplatin, 5-fluorouracil, \pm docetaxel for larynx preservation. J Natl Cancer Inst 2015;108.
[12] Forastiere AA, Zhang Q, Weber RS, Maor MH, Goepfert H, Pajak TF, et al. Longterm results of RTOG 91-11: a comparison of three nonsurgical treatment strategies to preserve the larynx in patients with locally advanced larynx cancer. J Clin Oncol 2013;31:845-52.

[13] Haughey BH, Hinni ML, Salassa JR, Hayden RE, Grant DG, Rich JT, et al. Transoral laser microsurgery as primary treatment for advanced-stage oropharyngeal cancer: a United States multicenter study. Head Neck 2011;33:1683-94.

[14] Weinstein GS, O'Malley Jr BW, Cohen MA, Quon H. Transoral robotic surgery for advanced oropharyngeal carcinoma. Arch Otolaryngol Head Neck Surg 2010;136:1079-85.

[15] Rusthoven KE, Raben D, Chen C. Improved survival in patients with Stage IIIIV Head and neck cancer treated with radiotherapy as primary local treatment modality. Int J Radiat Oncol Biol Phys 2008;72:343-50.

[16] Lee NY, de Arruda FF, Puri DR, Wolden SL, Narayana A, Mechalakos J, et al A comparison of intensity-modulated radiation therapy and concomitant boost radiotherapy in the setting of concurrent chemotherapy for locally advanced oropharyngeal carcinoma. Int J Radiat Oncol Biol Phys 2009;66: $966-74$.

[17] Rich JT, Milov S, Lewis Jr JS, Thorstad WL, Adkins DR, Haughey BH. Transora laser microsurgery (TLM) $+/$ - adjuvant therapy for advanced stage oropharyngeal cancer: outcomes and prognostic factors. Laryngoscope 2009;119: 1709-19.

[18] Cohen MA, Weinstein GS, O'Malley Jr BW, Feldman M, Quon H. Transoral robotic surgery and human papillomavirus status: oncologic results. Head Neck 2011;33:573-80.

[19] Bossi P, Alfieri S. The benefit of a multidisciplinary approach to the patient treated with (chemo) radiation for head and neck cancer. Curr Treat Options Oncol 2016;17:53.

[20] Friedland PL, Bozic B, Dewar J, Kuan R, Meyer C, Phillips M. Impact of multidisciplinary team management in head and neck cancer patients. Br J Cancer 2011;104:1246-8.

[21] Tsai WC, Kung PT, Wang ST, Huang KH, Liu SA. Beneficial impact of multidisciplinary team management on the survival in different stages of ora cavity cancer patients: results of a nationwide cohort study in Taiwan. Oral Oncol 2015;51:105-11.

[22] Wheless SA, McKinney KA, Zanation AM. A prospective study of the clinical impact of a multidisciplinary head and neck tumor board. Otolaryngol Head Neck Surg 2010;143:650-4.

[23] Brunner M, Gore SM, Read RL, Alexander A, Mehta A, Elliot M, et al. Head and neck multidisciplinary team meetings: Effect on patient management. Head Neck 2015;37:1046-50.

[24] Bergamini C, Locati L, Bossi P, Granata R, Alfieri S, Resteghini C, et al. Does a multidisciplinary team approach in a tertiary referral centre impact on the initial management of head and neck cancer? Oral Oncol 2016;54: $54-7$.

[25] Patil RD, Meinzen-Derr JK, Hendricks BL, Patil YJ. Improving access and timeliness of care for veterans with head and neck squamous cell carcinoma: a multidisciplinary team's approach. Laryngoscope 2016;126:627-31.

[26] Alfieri S, Orlandi E, Bossi P. The case volume issue in head and neck oncology Curr Treat Options Oncol 2017;18:65.

[27] Popescu RA, Schäfer R, Califano R, Eckert R, Coleman R, Douillard JY, et al The current and future role of the medical oncologist in the professional care for cancer patients: a position paper by the European Society for Medical Oncology (ESMO). Ann Oncol 2014;25:9-15. Erratum in: Ann. Oncol. 25 (2014) 916.

[28] Morgan G, Lambertini M, Kourie HR, Amaral T, Argiles G, Banerjee S, et al Career opportunities and benefits for young oncologists in the European society for medical oncology (ESMO). ESMO Open 2016;1. e000107.

[29] Dittrich C, Kosty M, Jezdic S, Pyle D, Berardi R, Bergh J, et al. ESMO/ASCO recommendations for a global curriculum in medical oncology Edition 2016 ESMO Open 2016;1. e000097.

[30] Wuthrick EJ, Zhang Q Machtay M, Rosenthal DI, Nguyen-Tan PF, Fortin A et al. Institutional clinical trial accrual volume and survival of patients with head and neck cancer. J Clin Oncol 2015;33:156-64.

[31] Peters LJ, O'Sullivan B, Giralt J, Fitzgerald TJ, Trotti A, Bernier J, et al. Critical impact of radiotherapy protocol compliance and quality in the treatment of advanced head and neck cancer: results from TROG 02.02. J Clin Oncol 2010;28:2996-3001.

[32] Tree AC, Harding V, Bhangu A, Krishnasamy V, Morton D, Stebbing J, et al. The need for multidisciplinarity in specialist training to optimize future patient care. Nat Rev Clin Oncol 2017;14:508-17.

[33] Gatta G, Trama A, Capocaccia R. Epidemiology of rare cancers and inequalities in oncologic outcome. Rare Cancers Monography. Eur. J Surg Oncol 2017;S07487983:30685-6.

[34] Gatta G, Capocaccia R, Botta L, Mallone S, De Angelis R, Ardanaz E, et al, RARECAREnet working group. Burden and centralised treatment in Europe of rare tumours: results of RARECAREnet-a population-based study. Lancet Oncol 2017;18:1022-39. 